\title{
Comprehensive Response of Rubber to Climate Change in Hainan, China
}

\author{
Shaojun Liu ${ }^{1,2, *}$ \\ ${ }^{1}$ Hainan Institute of Meteorological Sciences, 570203 Haikou, China \\ ${ }^{2}$ Key Laboratory of South China Sea Meteorological Disaster Prevention and Mitigation of Hainan Province, Haikou 570203, China
}

\begin{abstract}
The comprehensive response of rubber to climate change is influenced by many factors and each factor presents complex information. In this study, the input-output model of these influencing factors and rubber production disaster loss change were established using the data envelopment analysis method to analyze the comprehensive response of rubber to climate change in Hainan Island. Accordingly, the subjective factors could be avoided and reduced and the objectivity of evaluation could be improved. Climate influencing factors, sensitivity, and adaptability were used as input factors in the model, while rubber yield loss was used as the output factor. The efficiency of loss was used to simulate the comprehensive response of rubber to climate change. Results show that the high response of rubber to climate change appeared in Changjiang, Ledong, Wuzhishan, Qiongzhong, Wanning, and Qionghai. Meanwhile, the low response appeared in Wenchang, Lingao, Chengmai, Baisha, Dongfang, Sanya, and Lingshui. This research provides scientific support for the rubber plantation and the designation of countermeasures against the effects of climate change in Hainan Island.
\end{abstract}

\section{Introduction}

The impact of climate change on agriculture is a global concern $^{[1]}$. Climate change has changed crop growth period, production, and tillage system, as well as the frequency and intensity of meteorological disasters ${ }^{[2]}$. This phenomenon also inevitably exerts severe influence on rubber plantation. Effectively understanding and predicting the influence of future climate change on rubber production depends on accurate quantification of the response of rubber to previous climate change ${ }^{[3]}$. Therefore, study on the comprehensive response of rubber to climate change must start with rubber production.

The response models of crop yield to climate change are categorized into mechanism models(such as

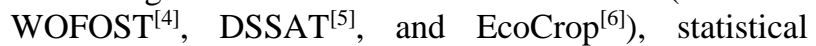
models (such as time series, cross-section, and panel models ${ }^{[7-9]}$ ), and economic models (such as Ricardian $\left.{ }^{[10]}\right)$. Currently, much research focuses on the response of agricultural system and one-seeded crop to climate change including wheat ${ }^{[11-15]}, \operatorname{cotton}^{[16]}, \operatorname{corn}^{[17]}$, rice ${ }^{[18]}$, and characteristic forestry and fruit ${ }^{[19]}$.However, research on the response evaluation of rubber to climate change is scarce. Some research has also evaluated the response of crops to climate change based on the model and statistical methods. However, these methods have many uncertainties in evaluation indexes and weights of different indexes ${ }^{[20,21]}$. The data envelopment analysis (DEA) method uses the single-input and single-output engineering efficiency concept ${ }^{[22]}$ as the basis without any weight assumption or function model, and solves the optimal weight in accordance with the actual data input and output by the decision-making unit; thus, this model avoids the influence of subjective factors and improves the objectivity of evaluation ${ }^{[23]}$.Thus, this study considered climate influencing factors, sensitivity, and adaptability as input factors and rubber production losses caused by disasters as the output factor. The process was conducted by selecting the evaluation indexes of the response of rubber to climate change with the Digital Elevation Model (DEM) as the tool to evaluate the response of rubber to climate change. This method aims to provide evidence for formulating the countermeasures of rubber against climate change scientifically.

\section{Data and Method}

\subsection{Study Area}

Hainan Island is the second largest island in China and covers an area of $3.43 \times 10^{4} \mathrm{~km}^{2}$. This island is located at $18.10^{\prime}$ to $20.10^{\prime} \mathrm{N}$ and $108.37^{\prime}$ to $111.03^{\prime} \mathrm{E}$ with a length of $290 \mathrm{~km}$ from northeast to southwest and a width of $180 \mathrm{~km}$ from northwest to southeast (Fig.1). In the tropical marine monsoon climate zone, Hainan Island is affected by the low-latitude tropical weather system and middle-high-latitude weather system. Thus, the climate in Hainan Island is complicated and unpredictable.

\footnotetext{
*Corresponding author: cdutlsj@163.com
} 
Hainan Island is one of the main natural rubber production areas in China. The actual plantation area of rubber in Hainan in 2011 reached $50.14 \times 10^{4} \mathrm{hm}^{2}$ with a total production of 372.3 thousand tons, accounting for $46.7 \%$ and $49.6 \%$ of the total production of rubber in China (datasource: Statistical Yearbook of Hainan Province 2012).

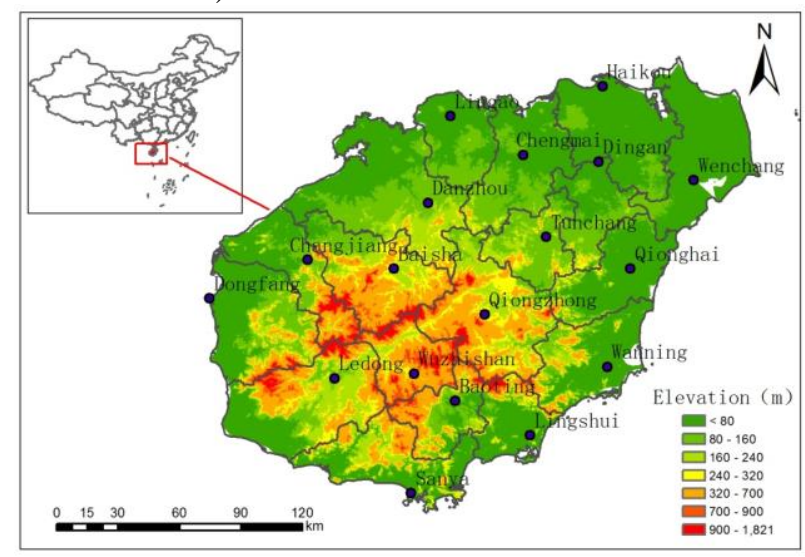

Fig.1 Location of the study area

\subsection{Data}

The following data were used in this study: daily data of 18 meteorological sites in Hainan Province during 1980 and 2010, including temperature, precipitation, wind speed, radiation, and other elements; the typhoon disaster that affected Hainan during 1981 and 2010 (source: Hainan Province Meteorological Bureau); rubber production data and per capita Gross Domestic Product ( GDP) data of different cities and counties in Hainan Province during 1990 and 2010 (source: Statistical Yearbook of Hainan Province); Hainan provincial boundary, and county line administrative zoning map (source: 1:4 million basic geographic information data provided by the National Fundamental Geographic Information website).

\subsection{Method}

The DEA model is an evaluation model of input-output operation efficiency. With any weight assumption in calculation, the DEA model avoids several subjective factors and is thus widely used in performance evaluation ${ }^{[24]}$. Research on the comprehensive response of rubber forests in Hainan Island to climate change starts mainly from the input-output perspective to construct the evaluation model using DEA, and then calculates the influential degree of climate change on rubber production.

DEA is an effective method to evaluate the efficiency of the multi-input and multi-output decision-making units (DMUs). The basic principle of DEA is to keep the output or input of DMU unchanged, determine the relatively effective production frontier through statistical data and mathematical programming, project DMUs to the production frontier of DEA, and compare the relative effectiveness of each DMU by comparing the deviation degree of DMU with the production frontier of $\mathrm{DEA}^{[22,25]}$.
The DEA method assumes that each input is associated with one or more outputs and the input has certain correlation with the output, but determining the display expression of the correlation is unnecessary [24]. Therefore, the difference in the response degree of rubber to climate change can be regarded as the rubber disaster efficiency output after using climate variability characteristic, amplitude, change rate, sensitivity, and adaptability as input conditions. The increased response degree will cause the increased disaster efficiency of rubber losses that lead to serious influence, and vice versa ${ }^{[23]}$. The evaluation on the comprehensive response of rubber to climate change is realized by the $C^{2} R-D E A$ model ${ }^{[26]}$. The specific algorithm is as follows:

$$
\left\{\begin{array}{l}
\min \left[\theta-\varepsilon\left(\hat{e}^{T} S^{-}+e^{T} S^{+}\right)\right] \\
\text {s.t. } \sum_{j=1}^{n} X_{j} \lambda_{j}+S^{-}=\theta X_{0} \\
\sum_{j=1}^{n} Y_{j} \lambda_{j}-S^{+}=Y_{0} \\
\lambda_{j} \geq 0, S^{-}, S^{+} \geq 0
\end{array}\right. \text {, }
$$

where $\theta$ is the comprehensive efficiencies of all DMUs of the DEA model; $\lambda_{j}(\mathrm{j}=1,2, \ldots, \mathrm{n})$ is the weight variable; $s^{-}$is the input slack variable; $s^{+}$is the output slack variable; $\quad \varepsilon$ is the non-Archimedes dimension(0.00001); $X_{0}$ and $Y_{0}$ are the input and output vectors of $\mathrm{DMU}_{0}$, respectively; and $Y_{j}$ is the output vector of the $j$ th $\mathrm{DMU}^{[26]}$.

Given the implication of the $\mathrm{C}^{2} \mathrm{R}$ model in the DEA method, $\theta$ ranges between 0 and 1. If $\theta$ of the evaluation unit is close to 1 , then the input-output operation level and production efficiency are high ${ }^{[24,26]}$. In the case of comprehensive response evaluation of rubber to climate change, when $\theta$ of the evaluation unit is close to 1 , then the influential degree of the climate change on the evaluation unit is high.

\subsection{Evaluation Factor}

The response degree of rubber to climate change refers to the degree of adverse influence of climate change (including climate variability and extreme climate events) on rubber production. The response degree of rubber to climate change is determined by the sensitivity of rubber to climate change and its comprehensive capability to adapt to climate change. In accordance with the requirements of the $C^{2} R$ model in DEA, the input-output factors of the model are constructed to evaluate the comprehensive response of rubber to climate change.

\subsubsection{Input Factors of the Model}

Climate influencing factors: temperature, precipitation, and solar radiation are the main meteorological factors affecting the latex production of rubber. They influence 
one another and act on the latex production of rubber with accumulative effect. The main soil nutrient elements, such as nitrogen, potassium, phosphorus, and magnesium, affect latex synthesis through photosynthesis as well as latex flow because of their impact on latex stability ${ }^{[27]}$. Rubber can tolerate the breeze but are non-resistant to the strong wind. Without considering the influence of the strong wind, the average wind speed of less than $1.0 \mathrm{~m} / \mathrm{s}$ exhibits a good effect on rubber tree growth; an average wind speed of between 1 and $1.9 \mathrm{~m} / \mathrm{s}$ has no effect on rubber tree growth; an average wind speed of between 2.0 and $2.9 \mathrm{~m} / \mathrm{s}$ shows an inhibition role on rubber tree growth and latex production; and an average wind speed of equal to or greater than $3.0 \mathrm{~m} / \mathrm{s}$ seriously restrains the growth and latex production of rubber trees ${ }^{[28]}$. The main natural factors affecting the survival and latex production of rubber trees in China are cold wave, low temperature, and typhoon ${ }^{[29-31]}$. Therefore, the climate tendency rates of temperature, wind speed, precipitation, and sunshine; and the intensity of typhoon and cold disaster are considered the climate influencing factors.

Sensitivity factor: sensitivity refers to the influential degree of the stimulating factors related to climate on the system, including adverse and beneficial effects ${ }^{[32]}$. As reported in [1, 33], the production reduction rate variation coefficient of rubber production can reflect the deviation extent of the production reduction rate from the average. The increased value indicates the wide inter-annual fluctuation, the less stable production reduction rate, and thus the increased sensitivity. The calculation formula of the variation coefficient is as follows (Formula (1)):

$$
C=\frac{\sqrt{\frac{1}{n-1} \sum_{1}^{n}\left(x_{i}-\bar{x}\right)^{2}}}{\bar{x}},(1)
$$

where $\bar{x}$ is the average rubber production reduction rate of all cities and counties; and $x_{\mathrm{i}}$ is the annual production reduction rate of natural rubber of the cities and counties.

Adaptability factor: adaptability is closely related to economy, technology, and resources ${ }^{[1,34]}$. As mentioned in[1], the per capita GDPs of different cities and the proportion of rubber plantation area in local total tillage area can be used as evaluation indexes of the adaptability of rubber. The reason is that the per capita GDPs of different cities can be considered the comprehensive capability to measure the adaptability of different cities to climate change, including improving varieties, adjusting tillage, applying advanced technology, buying chemical fertilizers and pesticides, and improving irrigation and infrastructure ${ }^{[1]}$. The proportion of the planting area represents the adaptability to the environment to some extent. A large planting area means a high adaptability to the environment ${ }^{[35]}$.

\subsubsection{Output Factors of the Model}

The inter-annual fluctuation of the statistical index of rubber disaster losses is significant because of the randomness characteristic of climate factor fluctuation and meteorological disaster occurrence. Thus, disaster loss data at a time point cannot be adopted. For this reason, the average of the rubber production losses caused by disasters in several years was used in the present study ${ }^{[23]}$. The rubber production losses caused by disasters represent the degree of adverse influence of climate change on rubber production. The trend production was calculated by the moving average in 5 years and the relative meteorological production was calculated by Formula (2). A negative value indicates the production reduction rate. The rubber production reduction rate was selected as the degree of rubber production losses caused by disasters.

$$
\mathrm{x}=\left(\mathrm{y}-\mathrm{y}_{\mathrm{t}}\right) / \mathrm{y}_{\mathrm{t}},(2)
$$

where $\mathrm{x}$ is the degree of rubber production losses caused by disasters; $\mathrm{y}$ is the actual unit production $\left(\mathrm{kg} / \mathrm{hm}^{2}\right)$; and $y_{t}$ is the trend unit production $\left(\mathrm{kg} / \mathrm{hm}^{2}\right)$.

\subsection{Evaluation Index Standardization}

The evaluation on the comprehensive response of rubber to climate change contains several factors. To eliminate $t$ he difference between the dimension and order of magnit ude of the indexes, each factor was normalized by Formu la (3).

$$
G=0.5+0.5 \times \frac{J_{i}-\min _{j}}{\max _{i}-\min _{j}} \text { (3) }
$$

where $G$ is the standardization value of the index; $J_{i}$ isthe $i$ th index value; and $\max _{i}, \min _{j}$ are the maximum and minimum values in the index values, respectively ${ }^{[36]}$.

\section{Result}

\subsection{Climate Influencing Factors}

The climate tendency rate of temperature, wind speed, precipitation, and sunshine were calculated using the meteorological data from 1980 to 2010.

Rubber cold damage index: the number of days with the average temperature less than $5{ }^{\circ} \mathrm{C}$ and the number of days with over 10 consecutive rain days and average temperature less than $15^{\circ} \mathrm{C}$ during 1981 and 2010 were used as the evaluation indexes. To allow comparison among the indexes, the ratio of the annual average number of days of a city or county (the number of days with an average temperature of less than $5{ }^{\circ} \mathrm{C}$ and the number of days with over 10 consecutive rain days and an average temperature of less than $15^{\circ} \mathrm{C}$ ) to the annual average number of days in Hainan Island (the number of days with an average temperature of less than $5{ }^{\circ} \mathrm{C}$ and the number of days with over 10 consecutive rain days and an average temperature of less than $15^{\circ} \mathrm{C}$ ) was used as the index of cold damage.

Typhoon damage index: the number of times with the wind speed reaches or exceeds 10 levels $(24.5 \mathrm{~m} / \mathrm{s})$ during 1981 and 2010 was used as the evaluation index. 
The processing method was similar to that of the cold damage index.

The climate tendency rates of temperature, wind speed, precipitation, and sunshine; the cold damage index; and the typhoon damage index were normalized. As a result, the high value areas of normalized average temperature climate tendency rate in Hainan Island were located in Sanya, Ledong, Changjiang, Wuzhishan, and Qiongzhong; the low value areas were in Chengmai, Ding'an, and Wenchang (Fig. 2a). The high value areas of normalized precipitation climate tendency rate were located in Wenchang, Sanya, Lingshui, Baoting, and Wuzhishan; the low value areas were in Qiongzhong (Fig. 2b). The high value areas of normalized sunshine climate tendency rate were located in Dongfang, Danzhou, Baisha, Wuzhishan, Tunchang, Qiongzhong, and Ding'an; the low value areas were in Haikou, Lingshui, and Chengmai (Fig. 2c). The high value areas of normalized wind speed climate tendency rate were located in Haikou, Chengmai, Ding'an, Wenchang, and Sanya; the low value areas were in Wuzhishan, Qiongzhong, and Baoting (Fig. 2d). The high value areas of normalized rubber cold damage index were located in Baisha, Qiongzhong, and Wuzhishan; the low value areas were in Dongfang, Changjiang, Haikou, Wenchang, Ding'an, Qionghai, Wanning, Lingshui, and Sanya (Fig. $3 e$ ). The high value areas of normalized typhoon damage index were located in Dongfang, Ledong, Sanya, Haikou, Wenchang, and Wanning; the low value areas were in Tunchang and Wuzhishan(Fig. 2f).
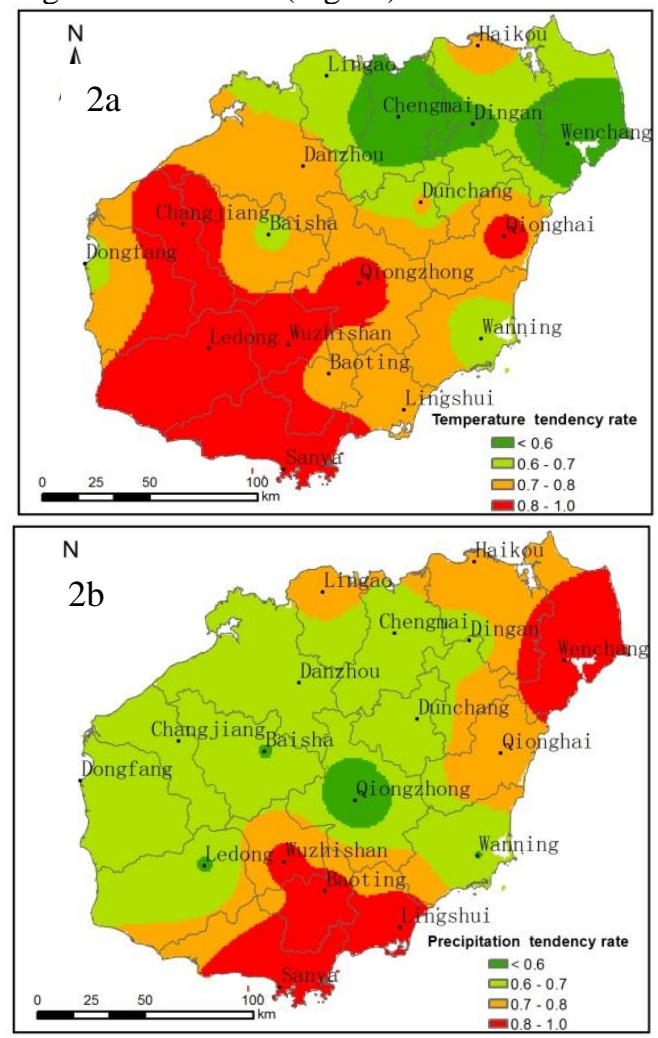
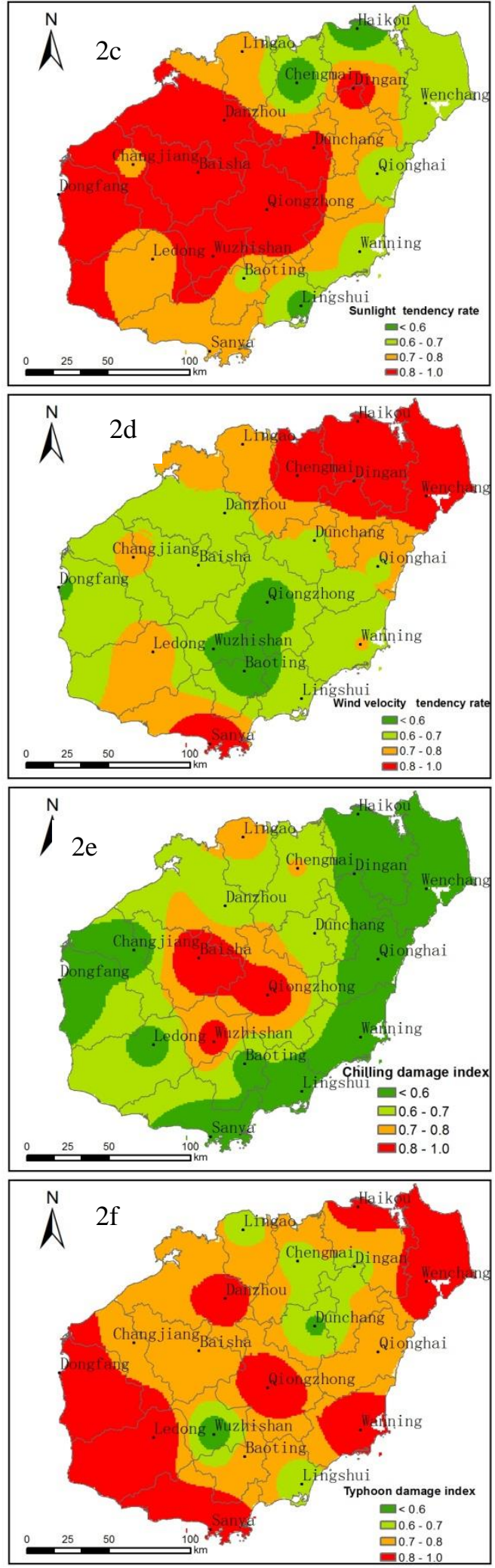

Fig.2 Climate indexes of rubber vulnerability assessment

\subsection{Sensitivity Factor}

Sensitivity factor embodies the deviation degree of the natural rubber production in a region from the average and indirectly reflects the fluctuation of the production under the influence of climate factors and environmental conditions. A large coefficient means a strong influence 
of the environment on the production. The sensitivity factor of rubber can be obtained after normalizing the variation coefficient of rubber production. As shown inFig.3, the sensitivity factors in Qiongzhong and Wanning were high, followed by those in Haikou, Ding'an, Wenchang, and Dongfang, and those in other cities with the lowest values.

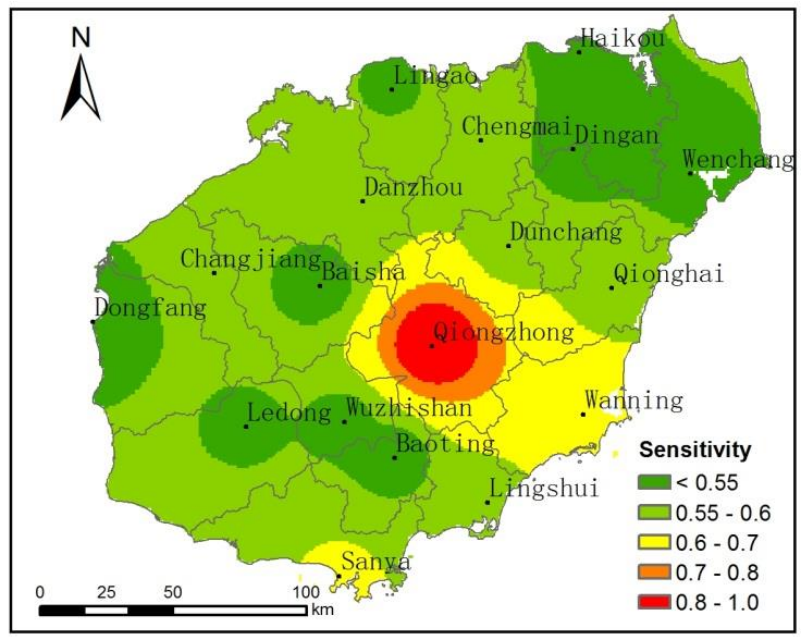

Fig.3 Sensitivity of rubber vulnerability assessment

\subsection{Adaptability Factor}
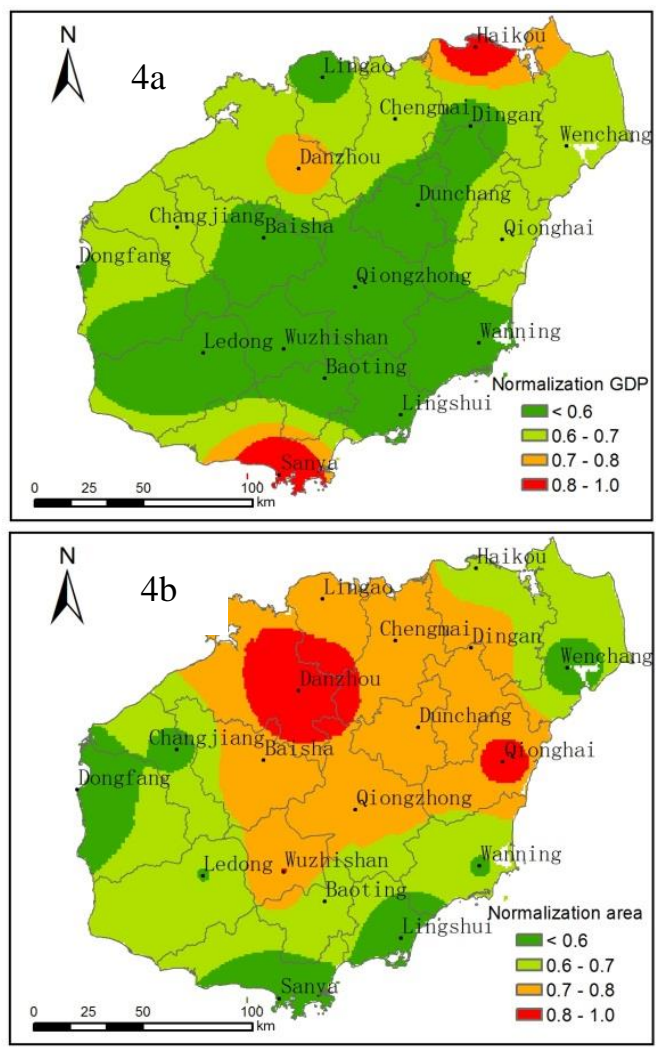

Fig.4 Adaptability of rubber vulnerability assessment

The per capita GDPs in different places and the proportion of rubber plantation area in the local tillage area were used to represent the adaptability of rubber. The two factors were normalized. Among them, the high value areas of normalized per capita GDP were located in Haikou and Sanya and the low value areas were in Ding'an, Tunchang, Baisha, Qiongzhong, Wanning, Ledong, Wuzhishan, Baoting, and Lingshui (Fig.4a). The high value areas of the proportion of rubber plantation area were located in Ding'an, Tunchang, Baisha, Qiongzhong, Wanning, Ledong, Wuzhi shan, Baoting, and Lingshui and the low value areas were in Dongfang, Wenchang, Lingshui, and Sanya (Fig.4b).

\subsection{Rubber Production Disaster Loss Factor}

The disaster degree of rubber production in Hainan Island showed a cauldron-shaped trend, which was closely related to the planting conditions and severe weather in Hainan Island. The high value areas of disaster loss were located in Wenchang, Lingshui, Sanya, Dongfang, and Changjiang; the low value areas were in Wuzhishan, Baoting, and Baisha in middle Hainan Island, and Danzhou in west Hainan Island (Fig.5).

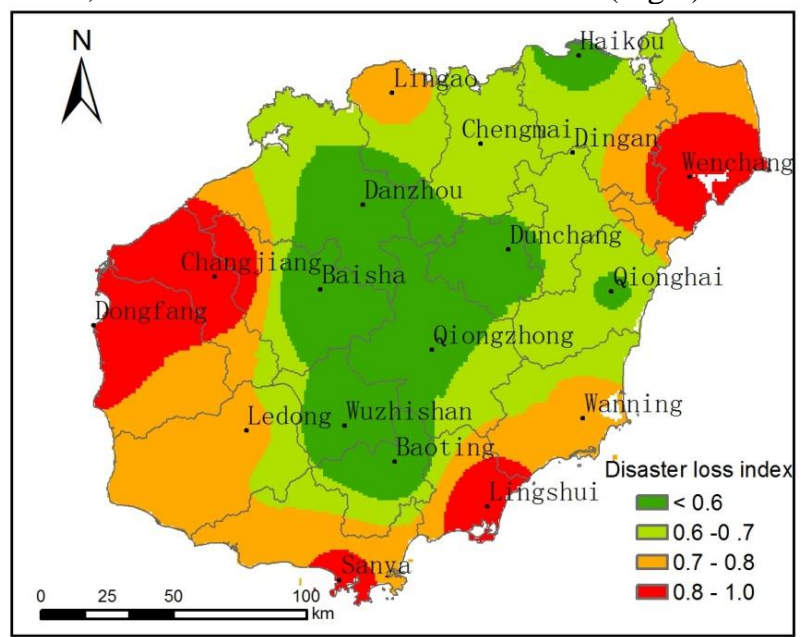

Fig.5 Disaster loss index of rubber

\subsection{Comprehensive Response of Rubber to Climate Change}

Climate influencing factors, sensitivity, and adaptability were used as input factors, and disaster loss degree of rubber production in Hainan Island was used as the output factor. They were substituted into the $C^{2} R$ model in MaxDEA software to calculate the disaster efficiency of rubber. The disaster efficiency of rubber represents the response degree of rubber to climate change. Overall, the rubber disaster efficiency in Hainan Island was high with an average value of 0.815 . The overall pattern of the response degree showed a mountain shape. The response degree was classified according to the meaning of $\theta$ in the DEA model: $0.85 \leq \theta \leq 1.0$, high response area; $0.75 \leq \theta<0.85$, middle response area; and $0<\theta<7.5$, low response area. The hierarchical distribution map of the response of rubber to climate change was thus obtained (Fig.6). As shown in the figure, the high response areas of rubber forests in Hainan Island were located in Changjiang, Ledong, Wuzhishan, Qiongzhong, Wanning, and Qionghai; the low response areas were located in 
Wenchang, Lingao, Chengmai, Baisha, Dongfang, Sanya, and Lingshui. Others were the middle response areas.

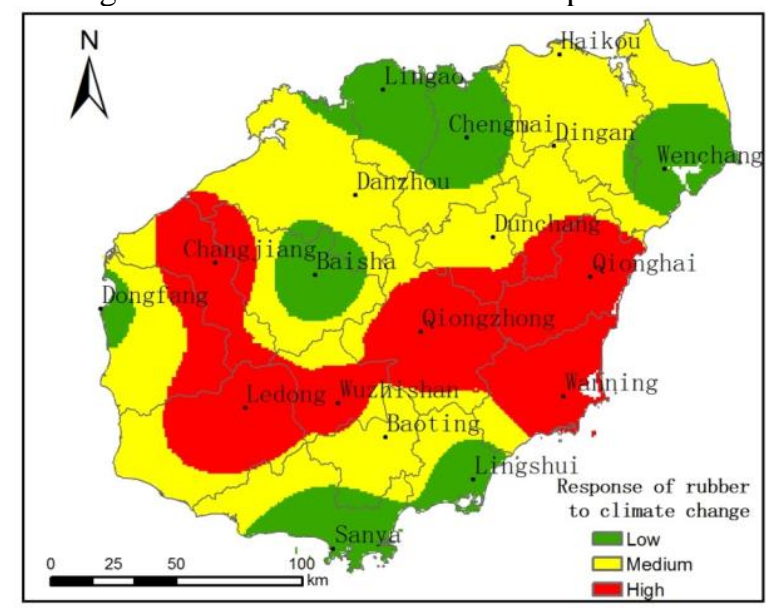

Fig.6 Response of rubber to climate change in Hainan Island

The analysis of the comprehensive response of rubber to climate change from the input-output perspective shows that the influence of climate change to rubber production is the combination of climate, sensitivity, and adaptability factors rather than a single factor. For example, in the high response area of Changjiang, temperature, sunshine, and typhoon were the influential factors of climate that occur frequently; and wind speed, precipitation, and cold disaster occurred less frequently. The sensitivity and adaptability factors were relatively low, but the disaster loss degree was relatively high. Thus, the response efficiency calculated by the model was high. In the low response area of Baisha, sunshine and cold disaster were the influential factors of climate that occur frequently; and temperature, wind speed, precipitation, and typhoon occurred less frequently. The sensitivity was low. The normalized per capita GDP was low but the normalized rubber area proportion was relatively high in the adaptability factor. Thus, the response efficiency calculated by the model was low. This result indicates that the evaluation model on the comprehensive response of rubber to climate change based on DEA exhibited good analysis effect.

\section{Discussion}

(1) The influence of climate change on rubber is a complicated process and climate change contains complex information ${ }^{[29]}$, thereby inevitably increasing the sensitivity difficulty of rubber to climate change. The comprehensive response of rubber to climate change is affected by the combined action of climate variability characteristics, amplitude, and rate of change as well as its sensitivity and adaptability. The response degree is different under different factors. Thus, the response degree cannot be expressed by a specific formula. The evaluation index weights on the response of crops to climate change and the constructed functions by predecessors are subjective with certain difficulty in revealing the response degrees caused by different factors. Thus, the DEA method is introduced. This method does not need any weight assumption, which avoids the subjective factor and objectively reflects the comprehensive response degree of rubber to climate change.

(2) Rubber is a typical tropical rain forest tree and is strict in meteorological conditions and sensitive to their changes ${ }^{[37]}$. Under the background of climate change, evaluating the comprehensive response of rubber to climate change in the major rubber production areas is necessary. Exploring appropriate evaluation index system, identifying the response difference of rubber production areas to climate change, and developing relevant measures can improve the capability of rubber to adapt to climate change. For example, the following tasks must be implemented to expand the rubber plantation area in the low response areas: adjust the rubber plantation area in the high response areas, strengthen the cultivation and promotion of improved varieties with wind resistance and high yield, improve the insurance awareness of farmers, and increase their capability to cope with huge disasters and risks ${ }^{[35]}$.

(3) If condition permits, then the evaluation on the response of rubber can be conducted in different growth periods and the comprehensive evaluation on the comprehensive response of rubber can be performed in the entire growth period. The reason is that the different requirements of rubber on climate conditions in different phenological periods and its different response degrees to climate change in different periods caused by the difference in climate time distribution must all be considered.

\section{Conclusion}

(1) The comprehensive response of rubber to climate change is affected by many factors. This study uses climate influencing factors (climate tendency rates of temperature, wind speed, precipitation and sunshine, and frequencies of cold disaster and typhoon), sensitivity factor, and adaptability factor (per capita GDP and proportion of rubber plantation area in local total tillage area) to establish the input-output model of the influential factors and rubber production disaster loss change by the DEA method. The influence efficiency, which indirectly reflects the response of rubber to climate change, is used to evaluate the comprehensive response of Hainan rubber to climate change. The method decreases the loss of information contained by the factors participating in evaluation and the influence of subjective factors to some extent and retains the information provided by the input and output variables at the extreme. This condition provides a new idea and method to evaluate the comprehensive response of rubber to climate change.

(2) Using the DEA model to evaluate the comprehensive response of rubber to climate change can reveal the influence of climate factors on rubber production accurately. Overall, the high response area of Hainan rubber to climate change shows a mountain shape. The response of Hainan rubber to climate change has strong spatial heterogeneity. Hainan rubber is affected mainly by the combination of climate 
influencing factors, sensitivity, and adaptability. Thus, the layout of the natural rubber industrial area can be optimized on the basis of the evaluation results.

The change of rubber production is affected by various factors, such as climate influencing factors, management technology, policy, and social economy. However, this study attributes the disaster loss change of rubber production to the influence of climate change and ignores other factors. This approach will inevitably lead to the deviation between the evaluation on the response of rubber to climate change and the actual situation. This point needs further improvement.

\section{Acknowledgments}

The research work was supported by the National Natural Science Foundation of China under Grant Nos. 41765007,41465005, 41265007, and 41675113, and the Natural Science Foundation of Hainan Provincial under Grant Nos. 20154172 and 409005. The writers are grateful for the support.

\section{References}

1. F. Sun, X. Yang, E.d. Lin,Sci. Agri. Sin. 38, 7 (2005)

2. L.Y. Xie, Y. Li, F.K. Qian , Chin. Popul. Res. Environ. 24, 6 (2014)

3. D.P. Xiao, F.L. Tao, Y.J. Shen, Chin. J. Eco. Agri. 22, 9 (2014)

4. Y. Curnel, A.J. De Wit, G. Duveiller, P. Defourny, Agric. Forest Meteo. 151, 13 (2011)

5. P.K. Thornton, P.G. Jones, G. Alagarswamy, J. Andresen, Glob. Environ. Change 19, 12 (2009)

6. J. Ramirez-Villegas, A. Jarvis, P. Läderach, Agric. Fores. Meteo. 170,12 (2013)

7. W. Schlenker, M.J. Roberts, Proc. Natl. Academ. Sci. 106, 5 (2009)

8. W.J. Shi, F.L. Tao, Sci. Agri. Sin. 47, 10 (2014)

9. W.J. Shi, F.L. Tao, Z. Zhang, Acta Geo. Sin. 67, 10 (2012)

10. S.N. Seo, R. Mendelsohn, Agric. Econo. 38, 15 (2008)

11. Nicholls N, Nature, 387, 2 (1997)

12. L. Z. You, M.W. Rosegrant, S. Wood, Agric. Fores. Meteo. 149, 6 (2009)

13. J.X. Zhang, J.P.Yan, J. Arid Land Res. Environ, 17, $6(2003)$

14. L.Q. Dai, C.Q. Li, R.J. Wei , Arid Zone Res. 28, 7( 2011)

15. H. Ju, W. Xiong, Y.L. Xu , Ecol. Environ. 17, 4 (2008)

16. W.D. Fu, Y.L. Yao, W.Y. Mao, Arid Zone Res. 26, 7 (2009)

17. H.Li, F.M.Yao, J.H. Zhang, Chin. J. Agromet. 35, 6 (2014)
18. Z. Zhu, The characteristics of rice production response to climate change in Jiangsu province (Master thesis of Nanjing University of Information Science and Technology, 2013)

19. J.Q. Liu, H.Wahap, A. Hasm, Acta Geo.Sin. 68,13(2013)

20. W.J. Shi, F.L. Tao, Z. Zhang. J. Geo. Sci. 23,10 (2013)

21. F.M. Yao, P.C. Qin, J.H. Zhang, Chin. Sci. Bull. 56, 9 (2011)

22. Z.X. Ma, Data envelopment analysis model and methods (Beijing,Science press,2010)

23. Y. Liu, J.Y. Huang, L. Ma, Geo. Res. 29,10 (2010)

24. S.J. Liu, J.H. Zhang, M.J. Zhang, J. Nat. Disa, 23, 8 (2014)

25. X.S.Wang, Z.X. Guo, J. Nat. Res. 29, 12 (2014)

26. Q.L. Wei, Data envelopment analysis (Beijing, Science press, 2004)

27. G.Y. Li, Q.B.Wang, Y.Y. Li , Chin. J. Eco. 33, 8 (2014)

28. Chinese Academy of tropical agriculture sciences,South China University of Tropical Agriculture. China tropical crop cultivation science (Beijing, China Agriculture Press Chinese, 1998)

29. A.L. Jiang, Trop. Geo. 23, 4 (2003)

30. A.L. Jiang, Acta Geo. Sin. 52, 9 (1997)

31. K. He, Z.D. Huang, The northern tropical rubber tree cultivation (Guangdong,Guangdong Science and Technology Press, 1987)

32. L.Yan, J.Q. Zhang, C.Y. Wang, Chin. J. Eco. Agric. 20, 7 (2012)

33. X.Yang, F.Sun, E.D. Lin, J. Nat. Disa. 13, 5 (2004)

34. R.T. Watson. Climate Change 2001:Synthesis Report (UK, Cambridge University Press, 2001)

35. X.G. Liu, H.J. Zhang, G.H. Li, J. Nat. Disa. 21, 7 (2012)

36. H.P. Zou, C.Y. Wang, J.H. Zhang, J. Nat. Disa. 22, 5 (2013)

37. Y.Q. Guo, R. Zhang, J. Yunnan Trop. Crops Sci.\& Tech. 4, 4 (1980) 